\title{
New option for management of HIV-I infection in treatment-naive patients: once-daily, fixed-dose combination of rilpivirine-emtricitabine-tenofovir
}

This article was published in the following Dove Press journal:

HIVIAIDS - Research and Palliative Care

26 April 2012

Number of times this article has been viewed

\author{
Nimish Patel' \\ Christopher D Miller ${ }^{1,2}$ \\ 'Albany College of Pharmacy and \\ Health Sciences, ${ }^{2}$ Albany Medical \\ Center Division of HIV Medicine, \\ Albany, NY, USA
}

Correspondence: Nimish Patel Albany College of Pharmacy and Health Sciences, 106 New Scotland Avenue, Albany, NY I2208, USA

$\mathrm{Tel}+\mathrm{I} 5186947159$

Fax +I 5186947062

Email nimish.patel@acphs.edu

\begin{abstract}
Fixed-dose combination tablets have become an important therapy option for patients infected with the human immunodeficiency virus. Fixed-dose combination rilpivirine-tenofoviremtricitabine is a recently approved therapy option that has been extensively studied within the treatment-naïve population. When compared with efavirenz-based therapy, improved tolerability with rilpivirine-based therapy was balanced by higher rates of virologic failure to provide similar overall efficacy rates within the intention-to-treat analysis. As a result, providers will need to balance the potential for improved tolerability with fixed-dose combination rilpivirine-tenofoviremtricitabine against a higher potential for virologic failure, particularly among patients with baseline viral loads above 100,000 copies/mL. Current treatment guidelines have recommended that fixed-dose combination rilpivirine-tenofovir-emtricitabine be an alternative therapy option for treatment-naïve patients and advise caution in those patients with high viral loads at baseline. Similar to other non-nucleoside reverse transcriptase inhibitor-based regimens, there are a number of drug interaction concerns with fixed-dose combination rilpivirine-tenofovir-emtricitabine that will necessitate monitoring and, in some cases, appropriate management. Additionally, the emergence of drug resistance to fixed-dose combination rilpivirine-tenofovir-emtricitabine has been well documented in clinical studies and close attention will be necessary in order to protect current and future therapy options. Overall, fixed-dose combination rilpivirine-tenofoviremtricitabine is poised to provide an important therapy option for patients when appropriately applied.
\end{abstract}

Keywords: rilpivirine, human immunodeficiency virus, antiretroviral, tenofovir, emtricitabine

\section{Introduction}

Antiretroviral therapy has evolved over the past two decades into a highly efficacious, well tolerated, and conveniently dosed group of therapies. Clinical studies of treatmentnaïve patients demonstrate virologic suppression rates above $80 \%$ for many of the new treatment regimens. ${ }^{1-3}$ In order to meet and maintain these high virologic suppression rates, strict medication adherence is necessary. ${ }^{4,5}$ Combination products, particularly among the nucleoside reverse transcriptase inhibitor class of agents, have become a standard of care to reduce pill burden and potentially improve adherence. ${ }^{6,7}$ However, since antiretroviral therapy is composed of a minimum of three active agents, patients may still require the administration of multiple tablets, regardless of the availability of nucleoside reverse transcriptase inhibitor combination tablets. This has changed with the creation of single-tablet regimens. The fixed-dose combination of efavirenz-tenofovir-emtricitabine was the first available daily-dosed single tablet that provided a complete antiretroviral regimen. ${ }^{8,9}$ Since its approval, fixed-dose combination efavirenz-tenofovir-emtricitabine 
has become an attractive and highly prescribed therapy for treatment-naïve patients due to strong efficacy data, good tolerability, and its low pill burden. ${ }^{6,7,10,11}$ The development of additional single-tablet regimens continues to be sought as a result of concerns about toxicity, resistance, and teratogenicity risks with the efavirenz component of fixed-dose combination efavirenz-tenofovir-emtricitabine. ${ }^{12-14}$ Fixed-dose combination rilpivirine-tenofovir-emtricitabine represents a more recently approved single-tablet regimen. ${ }^{15}$ Similar to fixed-dose combination efavirenz-tenofovir-emtricitabine, fixed-dose combination rilpivirine-tenofovir-emtricitabine contains tenofovir and emtricitabine, while the efavirenz component is replaced by rilpivirine. To date, fixed-dose combination rilpivirine-tenofovir-emtricitabine has demonstrated strong efficacy data and good tolerability and is poised to provide a valuable therapy option for human immunodeficiency virus (HIV)-infected patients. ${ }^{1,3}$ This paper reviews the efficacy, pharmacokinetics, tolerability, drug interaction potential, and resistance concerns with fixed-dose combination rilpivirine-tenofovir-emtricitabine. Given that the rilpivirine component of this fixed-dose combination is most recently available, we discuss this agent in more detail in the context of a fixed-dose combination.

\section{Pharmacology}

Fixed-dose combination rilpivirine-tenofovir-emtricitabine is composed of three active antiretroviral agents, ie, rilpivirine $25 \mathrm{mg}$ (rilpivirine hydrochloride $27.5 \mathrm{mg}$ ), tenofovir disoproxil fumarate $300 \mathrm{mg}$ (tenofovir disproxil $245 \mathrm{mg}$ ), and emtricitabine $200 \mathrm{mg} .{ }^{16}$ Dosage and administration characteristics of this fixed-dose combination are shown in Table $1 .{ }^{17}$ Consistent with current therapy recommendations for treatment-naïve patients, this combination spans two major classes of antiretroviral agents. ${ }^{6,7}$ Rilpivirine is a diarylpyrimidine non-nucleoside reverse transcriptase inhibitor (NNRTI). ${ }^{18}$ Similar to other NNRTIs, rilpivirine inhibits viral DNA polymerization by binding to the hydrophobic pocket near the active site of reverse transcriptase. ${ }^{19}$ As a diarylpyrimidine NNRTI, rilpirivine is commonly referred to as a "next-generation" or "second-generation" NNRTI. Agents from this subclass possess a more flexible dihedral angle between the aniline ring and cyanovinyl moiety when compared with first-generation agents. ${ }^{7,20}$ This characteristic allows for binding to multiple modes within the highly flexible NNRTI binding site of reverse transcriptase and potentially leads to an improved resistance profile, although currently available clinical study data with rilpivirine have placed this advantage into question., ${ }^{1,3}$
Table I Administration and place in therapy ${ }^{17,71}$

\begin{tabular}{|c|c|}
\hline Dosage and administration & $\begin{array}{l}\text { - One tablet by mouth once daily } \\
\text { - Administer with a meal }\end{array}$ \\
\hline Dosage form & $\begin{array}{l}\text { Tablets (purplish-pink, capsule-shaped, } \\
\text { film-coated) }\end{array}$ \\
\hline Strengths & $\begin{array}{l}\text { Each tablet contains: } \\
\text { - } 25 \mathrm{mg} \text { of rilpivirine } \\
\text { - } 300 \mathrm{mg} \text { of TDF } \\
\text { - } 200 \mathrm{mg} \text { FTC }\end{array}$ \\
\hline Dosage adjustments & $\begin{array}{l}\text { Renal impairment: } \\
\text { - Do not use in patients with moderate } \\
\text { to severe renal impairment (creatinine } \\
\text { clearance }<50 \mathrm{~mL} \text { per minute). Separate } \\
\text { agents to accommodate dosage } \\
\text { adjustments necessary for TDF and FTC } \\
\text { Hepatic impairment: } \\
\text { - No adjustment required for patients with } \\
\text { mild or moderate hepatic impairment } \\
\text { (Child-Pugh Classes A and B) } \\
\text { - Not been studied in patients with severe } \\
\text { hepatic impairment (Child-Pugh Class C) }\end{array}$ \\
\hline $\begin{array}{l}\text { Important adverse effect } \\
\text { concerns that may } \\
\text { require monitoring }\end{array}$ & $\begin{array}{l}\text { Rash, renal dysfunction, losses in bone } \\
\text { mineral density, depressive disorders }\end{array}$ \\
\hline $\begin{array}{l}\text { Recommended place in } \\
\text { therapy per antiretroviral } \\
\text { treatment guidelines }\end{array}$ & $\begin{array}{l}\text { Alternative therapy option for NNRTI- } \\
\text { based antiretroviral therapy } \\
\text { - Caution recommended in patients with } \\
\text { baseline viral load }>100,000 \text { copies } / \mathrm{mL}\end{array}$ \\
\hline
\end{tabular}

Abbreviations: NNRTI, non-nucleoside reverse transcriptase inhibitor; FTC, emtricitabine; TDF, tenofovir disoproxil fumarate.

Tenofovir disoproxil fumarate is a nucleotide reverse transcriptase inhibitor. ${ }^{21}$ Following absorption, this compound is converted to tenofovir by diester hydrolysis and then subsequently phosphorylated to form tenofovir diphosphate. ${ }^{22}$ The addition of a phosphate group is the key structural difference between a nucleoside and nucleotide, although clinically these drug classes are considered to be largely equivalent. Tenofovir diphosphate inhibits HIV reverse transcriptase by competing with the natural substrate deoxyadenosine $5^{\prime}$-triphosphate for incorporation into viral DNA, leading to termination of viral DNA growth. ${ }^{22}$ Tenofovir is active against both HIV-1 and HIV-2 and also has activity against hepatitis $B$ virus. ${ }^{23,24}$

Emtricitabine is a nucleoside reverse transcriptase inhibitor. ${ }^{25}$ Emtricitabine is the (-) enantiomer of a thio analog of cytidine and, unlike other cytidine analogs, has a fluorine in the 5-position. ${ }^{26}$ The molecular structure of emtricitabine is highly similar to another antiretroviral agent, lamivudine. In clinical practice, these agents are commonly considered to be interchangeable in terms of efficacy and safety. ${ }^{6,727}$ Emtricitabine also has activity against HIV-1, HIV-2, and hepatitis B virus..$^{25,28}$ 


\section{Clinical pharmacokinetics}

The pharmacokinetic characteristics of each of the components of fixed-dose combination rilpivirine-tenofovir-emtricitabine are presented in Table 2. ${ }^{21,22,29-32}$ The pharmacokinetics of each agent supports once-daily dosing. The plasma elimination half-lives are approximated as follows: rilpivirine 48 hours; tenofovir 17 hours; and emtricitabine 10 hours..$^{22,29-31,33}$ The intracellular elimination half-lives of tenofovir and emtricitabine are significantly longer and approximated as follows: tenofovir 150 hours and emtricitabine 39 hours. ${ }^{31}$ As a result, this fixed-dose combination can be conveniently dosed on a once-daily basis.

When fixed-dose combination rilpivirine-tenofoviremtricitabine was administered with food (400 kcal and 13 grams of fat) in healthy subjects, rilpivirine, tenofovir, and emtricitabine exposures were bioequivalent to the administration of the individual tablets. When the fixed-dose combination was administered to healthy subjects in the fasted state, rilpivirine and emtricitabine concentrations were approximately $25 \%$ higher when compared with administration of the individual tablets. ${ }^{16,34}$ As a result of these differences, it is recommended that fixed-dose combination rilpivirine-tenofovir-emtricitabine be administered with a meal. ${ }^{16}$ However, no specific meal type has been recommended. Rilpivirine should not be administered with a high protein meal, such as protein-rich nutritional drinks, because exposures are reduced by $50 \%{ }^{17,35}$ The solubility and subsequent systemic absorption of rilpivirine is $\mathrm{pH}$-dependent, as demonstrated by a significantly increased bioavailability when given in an acidic environment. When coadministered with acid suppressants, significant reductions in drug absorption were observed. ${ }^{36}$ As a result, fixed-dose combination rilpivirine-tenofoviremtricitabine should not be coadministered with proton pump inhibitors. $\mathrm{H} 2$ antagonists may be coadministered with this fixed-dose combination if spaced appropriately, as discussed in the drug interaction section. ${ }^{16}$

The components of fixed-dose combination rilpivirinetenofovir-emtricitabine undergo different routes of elimination. Rilpivirine primarily undergoes oxidative metabolism through cytochrome P450 (CYP) 3A, while both tenofovir and emtricitabine are eliminated via a mixture of glomerular filtration and active tubular secretion. . $6,22,30,31^{2}$ Both tenofovir and emtricitabine require dosage adjustment in patients with a creatinine clearance below $50 \mathrm{~mL}$ per minute while rilpivirine does not. ${ }^{16,21,25,37}$ Due to this disparity, fixed-dose combination rilpivirine-tenofovir-emtricitabine should not be administered to patients with a creatinine clearance below $50 \mathrm{~mL}$ per minute and the components should be separated to accommodate the necessary dosage adjustments. None of the components of this fixed-dose combination require dosage adjustment in patients with mild or moderate hepatic impairment. ${ }^{16}$ There are insufficient data about dosing and safety in patients with severe hepatic impairment.

\section{Single-tablet regimens}

Currently, there are two single-tablet regimen products that are commercially available, and the antiretroviral components are efavirenz-tenofovir-emtricitabine and rilpivirine-tenofovir-emtricitabine. Coformulation has significantly reduced the overall pill burden and improved the convenience of taking antiretroviral therapy. The majority of data regarding single-tablet regimens focus mainly on fixed-dose combination efavirenz-tenofovir-emtricitabine because it has been available since 2006. Nonetheless, the data available on fixed-dose combination efavirenz-tenofovir-emtricitabine are helpful in understanding the potential benefits of using a fixed-dose combination of rilpivirine-tenofoviremtricitabine.

Several studies have demonstrated that switching to fixed-dose combination efavirenz-tenofovir-emtricitabine was generally effective in maintaining virologic suppression in patients who had an undetectable viral load at the time of switching therapy. ${ }^{38,39}$ The proportion of patients achieving virologic suppression was not significantly different among patients receiving fixed-dose combination efavirenz-tenofoviremtricitabine compared with patients who received more complex antiretroviral regimens. ${ }^{39,40}$ These effects were seen out to 48 weeks in most studies and up to 96 weeks in one study. ${ }^{38}$ Given the potency of most antiretroviral agents, it is unsurprising that single-tablet regimen products achieve a similar frequency of virologic suppression compared with more complex antiretroviral regimens. Outcomes that may differentiate single-tablet regimen regimens from antiretroviral regimens with higher complexity include quality of life, adherence, patient preference, perceived medication regimen complexity, and tolerability.

In the ADONE study, health-related quality of life improved during the course of the study ${ }^{38}$ Specifically, the difference in the quality of life measure was significantly improved after 24 weeks of switching to fixed-dose combination efavirenztenofovir-emtricitabine compared with baseline. ${ }^{38}$ Based on these data, single-tablet regimens appear to be beneficial for improving quality of life. However, when comparing the quality of life of single-tablet regimen recipients with that of patients who remained on their original antiretroviral 
regimens, there may not be a meaningful change. ${ }^{39}$ In a trial of virologically suppressed HIV-infected patients randomized to either switch to fixed-dose combination efavirenz-tenofoviremtricitabine or maintain their current antiretroviral regimen, quality of life measures did improve from baseline in either group. ${ }^{39}$ However, changes in these quality of life measures did not differ significantly between groups. ${ }^{39}$

In the studies that have evaluated adherence, there appears to be a high proportion of patients adhering to single-tablet regimens through 48 weeks of therapy. ${ }^{38}$ There was also a higher preference for single-tablet regimens over original antiretroviral regimens, and patients perceived that singletablet regimens were easier to follow than more complex treatment regimens. ${ }^{39}$ The tolerability of fixed-dose combination regimens will depend heavily on the components of the single-tablet regimen. The studies that have been performed thus far have been with fixed-dose combination efavirenztenofovir-emtricitabine, and the majority of adverse effects were related to efavirenz use. ${ }^{38-40}$ While the overall proportion of adverse events was low, there did appear to be a higher proportion of psychiatric symptoms, nervous system symptoms, and skin/tissue disorders among single-tablet regimen recipients compared with patients who did not modify their antiretroviral regimen. ${ }^{39}$ For the rilpivirine-containing single-tablet regimen, it is unclear if the frequency of adverse events will differ.

One interesting outcome of single-tablet regimen medication use is hospitalizations. Compared with patients using antiretroviral regimens that contain two or more pills per day, single-tablet regimen recipients were more likely to achieve optimal adherence thresholds and the risk of hospitalization was $25 \%$ lower. $^{7}$ As more fixed-dose combination products emerge on the market, ancillary health outcomes, like hospitalizations, associated with single-tablet regimen use will need to be evaluated for each product. The majority of studies evaluating single-tablet regimen products have focused on populations that are either treatment-experienced or virologically suppressed and switching to a single-tablet regimen product. Outcomes for viremic patients who are initiating a single-tablet regimen product should be evaluated. Given that fixed-dose combination rilpivirine-tenofovir-emtricitabine is only approved for treatment-naïve individuals, the treatment outcomes of single-tablet regimens in treatment-naïve individuals will need to be assessed.

\section{Efficacy}

Rilpivirine has been evaluated in two Phase III studies, ie, ECHO (a Phase III randomized study of the efficacy of rilpivirine compared with efavirenz) and THRIVE (a randomized Phase III study of the efficacy of rilpivirine compared with efavirenz in HIV-infected patients using either tenofovir-emtricitabine, zidovudine-lamivudine, or abacavirlamivudine). ${ }^{1,3}$ In the ECHO study, patients were exclusively prescribed tenofovir-emtricitabine as the nucleoside reverse transcriptase inhibitor backbone. ${ }^{3}$ In the THRIVE study, providers could choose between tenofovir-emtricitabine, lamivudine-zidovudine, or abacavir-lamivudine for the nucleoside reverse transcriptase inhibitor backbone. ${ }^{1}$ Sixty percent of patients in the THRIVE trial were given tenofoviremtricitabine as their background nucleoside reverse transcriptase inhibitor regimen. ${ }^{1}$ For this reason, this review will first concentrate on the ECHO study because it exclusively studied a regimen consistent with the components of fixeddose combination rilpivirine-tenofovir-emtricitabine.

The ECHO trial was a randomized, double-blind, comparative study of rilpivirine versus efavirenz in treatment-naïve patients infected with HIV-1. ${ }^{3}$ The primary objective of the study was to show noninferiority between a rilpivirine-based and efavirenz-based antiretroviral regimen. The primary endpoint was the proportion of patients who achieved a viral load of $<50$ copies $/ \mathrm{mL}$ or maintained a viral load $<50$ copies $/ \mathrm{mL}$ after 48 weeks of treatment. Secondary endpoints included pharmacokinetics, safety, immune response, and emergence of drug resistance. Participants were individuals $\geq 18$ years of age who were infected with HIV-1, were treatment-naïve, and had a baseline viral load $>5000$ copies $/ \mathrm{mL}$. Randomization was stratified by baseline viral load $(\leq 100,000, \leq 500,000$, and $>500,000$ copies $/ \mathrm{mL}$ ).

After 1:1 randomization, the intention-to-treat analysis included 346 patients who were assigned to receive rilpivirine and 344 who received efavirenz-based therapy. At 48 weeks of treatment, $83 \%$ of patients in both groups had a confirmed virologic response, ie, a difference of $0.1 \%$ ( $95 \%$ confidence interval [CI] $0.1[-5.5 \%$ to $5.7 \%])$. Although the overall intention-to-treat outcomes were similar, the reasons for treatment failure differed between the treatment groups. More virologic failures for the efficacy endpoint (patients who never had virologic suppression or experienced virologic rebound) occurred with rilpivirine (38 patients [11\%] with rilpivirine versus 15 patients [4\%] with efavirenz). In contrast, more patients in the efavirenz group discontinued therapy and were considered a treatment failure due to adverse events. Specifically, 19 patients (7\%) in the efavirenz group discontinued therapy due to an adverse event as compared with six patients (2\%) in the rilpivirine group. The majority of adverse events leading to treatment discontinuation with 
efavirenz were central nervous system effects. In a sensitivity analysis that excluded patients who discontinued therapy for reasons other than virologic failure, response rates were $86 \%$ (287/333) and 94\% (285/303) for the rilpirivine and efavirenz groups, respectively (difference $-7.9 \%$, 95\% CI $-12.5 \%$ to $-3.2 \%$ ). The immunologic response was similar between the treatment groups, with a mean change in absolute CD4 count of 196 cells $/ \mu \mathrm{L}$ (95\% CI 179-212) for rilpivirine and 182 cells $/ \mu \mathrm{L}(95 \%$ CI 165-198) for efavirenz $(P=0.13)$.

For the purpose of the 96-week analysis, data from the ECHO and THRIVE studies were pooled. ${ }^{41}$ Although the majority of patients in this pooled analysis used tenofoviremtricitabine as their nucleoside reverse transcriptase inhibitor backbone regimen, there were patients using abacavir-lamivudine or zidovudine-lamivudine. The overall virologic response was $77.6 \%$ in both the rilpivirine and efavirenz treatment groups $[95 \% \mathrm{CI}(-4.4,4.4)]$. Immunologic response was also similar between groups and consistent with the 48-week ECHO data.

Again, although overall response rates were similar between groups, there were more virologic treatment failures in the rilpivirine group and more patients who discontinued therapy due to toxicity in the efavirenz group. For patients who entered the study with a baseline viral load $>100,000$ copies/mL, virologic failure was more common in the rilpivirine group. There was no difference in virologic failure shown for patients who entered the study with a baseline viral load $\leq 100,000$ copies $/ \mathrm{mL}$.

\section{Safety}

Overall, the components of fixed-dose combination rilpivirine-tenofovir-emtricitabine demonstrate a favorable safety profile. The safety of rilpivirine has largely been judged based upon its comparison with efavirenz in clinical study. ${ }^{1,3}$ As highlighted in the efficacy section, overall adverse event rates were lower with rilpivirine, and significantly fewer patients discontinued rilpirivine therapy due to toxicity when compared with efavirenz. The main driver for these differences was central nervous system toxicity, a known and relatively common side effect of efavirenz. ${ }^{1,3}$ Although rilpivirine still has some risk for central nervous system toxicity (including headache, depressive disorders, and insomnia) it occurs significantly less often. Another important difference displayed in the ECHO and THRIVE studies was the difference in lipid effects between rilpivirine and efavirenz. ${ }^{1,3}$ Previous data show that efavirenz can cause significant increases in low-density lipoprotein, high-density lipoprotein, and total cholesterol. ${ }^{42-44}$ In clinical study, the effects of rilpivirine on the serum lipid profile were minimal. Grade 2 or higher changes in lipids and triglycerides occurred in less than $6 \%$ of rilpivirine-treated patients. ${ }^{1,3}$ Grade 2 lipid changes occurred in roughly $10 \%-20 \%$ of efavirenz-treated patients. Liver function tests were similar between rilpivirine and efavirenz treatment, with $\leq 3 \%$ of patients experiencing a grade 3 or 4 elevation in aspartate transaminase or alanine transaminase. ${ }^{1,3,44}$ Five percent of rilpivirine-treated patients experienced a grade 1 elevation in serum creatinine as compared with $\leq 1 \%$ with efavirenz-based therapy. The mean change was $0.09 \mathrm{mg} / \mathrm{dL}$ (range $-0.20 \mathrm{mg} / \mathrm{dL}$ to $0.62 \mathrm{mg} / \mathrm{dL}$ ). When increases in serum creatinine occurred, they most commonly occurred during the first month of therapy and persisted for the entire 48 weeks of therapy, and no subjects discontinued therapy due to increases in serum creatinine. ${ }^{41}$ The mechanism of this effect remains unknown and the clinical impact appears to be minimal according to the available data.

Other side effects observed with rilpivirine in clinical study include nausea and rash, both of which occurred in $\leq 3 \%$ of patients. ${ }^{1,2,41}$ Rilpivirine is associated with a dosedependent increase in the QTc interval. ${ }^{25}$ At the approved dose of $25 \mathrm{mg}$, increases in the QTc interval appear to be minimal and are not expected to result in clinically adverse effects in patients without pre-existing cardiac conditions. At higher doses, the QTc prolongation effect is more pronounced and patients who are overdosed with rilpivirine should have electrocardiographic monitoring performed. ${ }^{25}$

The principal toxicity associated with use of tenofovir is nephrotoxicity. ${ }^{45}$ The most common form is proximal tubular toxicity characterized by electrolyte wasting and serum creatinine elevations. Fanconi syndrome and acute renal failure have been documented which, in some cases, have led to irreversible renal dysfunction. ${ }^{46-49}$ Clinical studies have shown the overall incidence of nephrotoxicity due to tenofovir to be $<1 \%$; however, small and gradual reductions in kidney function have more commonly been shown in clinical studies. ${ }^{50}$ Outside of the controlled setting of a clinical study, the true rates of tenofovir-induced nephrotoxicity appear to be higher. Numerous case reports and case series have been published to document this. ${ }^{46-48,51,52}$ Monitoring of renal function is recommended for patients who are prescribed a tenofovir-containing regimen. Particular attention should be paid to patients who have existing risk factors for tenofovir-induced nephrotoxicity. These risk factors include underlying renal dysfunction, older age, concurrent use of nephrotoxic medications, concurrent use of protease inhibitors, low body weight, and low CD4 count. ${ }^{53-56}$ 
Reductions in bone mineral density have also been documented in tenofovir-treated patients. Randomized clinical trials show that this effect occurs more commonly when patients are treated with tenofovir as compared with a regimen that does not contain tenofovir. ${ }^{57}$ As a result, it is recommended that consideration be given to assessing bone mineral density in tenofovir-treated patients with risk factors for osteoporosis or bone loss.

Emtricitabine has one of the strongest safety profiles when compared with other antiretroviral agents, and is generally considered to have minimal risk of toxicity. ${ }^{58,59}$ In clinical study, the most common adverse effects observed in antiretroviral regimens that included emtricitabine were headache, diarrhea, nausea, fatigue, dizziness, depression, insomnia, abnormal dreams, rash, abdominal pain, asthenia, increased cough, and rhinitis. ${ }^{60}$ However, it is important to note that it is difficult to discern whether these adverse effects were directly caused by emtricitabine or the coadministered antiretroviral agents. Hyperpigmentation and/or skin discoloration is a unique adverse effect that has been observed with emtricitabine but this effect occurs predominantly in children. ${ }^{61}$

Safety data with the combination product, fixed-dose combination rilpivirine-tenofovir-emtricitabine are limited, although expected to be similar to what is observed when the individual agents are administered. As a result, mild adverse effects that may occur include gastrointestinal toxicities (nausea, vomiting, diarrhea), headache, and insomnia. More concerning adverse effects that require patient monitoring may include rash, renal dysfunction, loss of bone mineral density, and depressive disorders. To date, there are no toxicity data to suggest that adverse effect risks with fixed-dose combination rilpivirine-tenofovir-emtricitabine are different to those of the individual agents.

\section{Resistance}

Resistance associated with tenofovir and emtricitabine will not be discussed in this paper and are reviewed elsewhere. ${ }^{62}$ The resistance profile of rilpivirine is unique relative to other NNRTIs because it exhibits in vitro activity against both wild-type and drug-resistant HIV-1 isolates. ${ }^{63}$ Rilpivirine displays potent activity against HIV-1strains that are resistant to older NNRTIs like efavirenz and nevirapine. ${ }^{6}$ At the present time, rilpivirine is only approved for use in patients that are treatment-naïve. However, the activity of rilpivirine against HIV-1 isolates with diminished activity against first-generation NNRTIs may still be important to consider due to transmitted resistance risks. Approximately
$5 \%-10 \%$ of treatment-naïve patients acquire HIV infection with transmitted drug resistance and these patients experience a higher frequency of virologic failure when compared with patients who are transmitted drug-sensitive virus. ${ }^{64,65}$ As a result, rilpivirine may be an attractive option for treatment-naïve patients with transmitted drug resistance to other NNRTIs.

In ECHO, there were some differences regarding virologic failure. ${ }^{3}$ Virologic failures with resistance occurred in $13 \%$ of patients in the rilpivirine treatment arm and $6 \%$ in the efavirenz group. ${ }^{3}$ There were 53 patients (40 in the rilpivirine group and 13 in the efavirenz group) who experienced virologic failure and had resistance data available at the time of failure. Among these patients, the frequency of virologic failure with any treatment-emergent NNRTI resistance-associated mutation was similar between rilpivirine and efavirenz groups ( $65 \%$ versus $62 \%$, respectively). ${ }^{3}$ The most common treatment-emergent NNRTI resistanceassociated mutation in the rilpivirine group was the E138K mutation (69\%), followed by K101E (19\%) and Y181C $(19 \%) .{ }^{3}$ There was a significant difference in the proportion of patients who experienced virologic failure with any treatment-emergent International AIDS Society-USA (IAS-USA) nucleoside/nucleotide reverse transcriptase inhibitor resistance-associated mutation. ${ }^{3}$ Specifically, the frequency of M184I was $71 \%$ and $25 \%$ in rilpivirine and efavirenz patients, respectively. ${ }^{3}$

In the THRIVE study, ${ }^{1}$ there appeared to be nearly a two-fold difference in the proportion of patients experiencing virologic failure with any treatment-emergent IAS-USA nucleoside/nucleotide reverse transcriptase inhibitor resistance-associated mutation $(64 \%$ of rilpivirine patients compared with $33 \%$ of efavirenz patients). ${ }^{1}$ Among patients in the rilpivirine group experiencing virologic failure, the most common treatment-emergent NNRTI resistanceassociated mutations were E138K (77\%), K101E (73\%), V189I (15\%), and H221Y (15\%). ${ }^{1}$ Among the IAS-USA nucleoside/nucleotide reverse transcriptase inhibitor resistance-associated mutations identified, the overall frequencies of the $\mathrm{M} 184 \mathrm{~V}$ and/or I mutations were $86 \%$ and $60 \%$ for patients in the rilpivirine and efavirenz groups, respectively, who experienced virologic failure. ${ }^{1}$

A major resistance concern regarding antiretroviral resistance to NNRTI agents is the issue of cross-resistance. Patients who experienced virologic failure in the rilpivirine groups of the ECHO and THRIVE studies with evidence of phenotypic resistance to rilpivirine had evidence of cross-resistance to efavirenz, etravirine, and nevirapine. ${ }^{1,3,66}$ Specifically, of the 
31 rilpivirine-treated patients who experienced virologic failure and developed phenotypic resistance to rilpivirine, the frequencies of cross-resistance to etravirine, efavirenz, and nevirapine were $90 \%, 87 \%$, and $45 \%$, respectively. ${ }^{3}$ Given that rilpivirine is a drug that is approved for treatment-naïve individuals, the issue of cross-resistance may deter its use in practice. Efavirenz, nevirapine, and etravirine have been used in patients who are treatment-experienced, and the antecedent use of rilpivirine in a treatment-naïve individual may limit the use of other NNRTI agents if virologic failure and phenotypic resistance occurs.

\section{Drug interactions}

Drug interactions with tenofovir and emtricitabine are described elsewhere. ${ }^{62}$ This review will focus on drug interactions associated with the rilpivirine component of fixed-dose combination rilpivirine-tenofovir-emtricitabine. Like most NNRTIs, rilpivirine is metabolized through the CYP isoenzyme system. It is both a substrate and inducer of CYP3A4. ${ }^{18,67}$ Rilpivirine is primarily metabolized by CYP 3A4. However, CYP2 C19, 1A2, and 2C8/9/10 are also involved. There are also some inductive effects associated with rilpivirine use $^{68}$ Specifically, rilpivirine is a moderate inducer of CYP 2C19 and 3A4 and has subtle induction effects on CYP $1 \mathrm{~A} 2$ and $2 \mathrm{~B} 6 .{ }^{68}$ As a result of these effects on $\mathrm{CYP}$ isoenzymes and the necessity for an acidic gastric $\mathrm{pH}$ for absorption, there are a number of drug interactions associated with the use of the rilpivirine component of fixed-dose combination tenofovir-emtricitabine-rilpivirine. Select drug interactions with rilpivirine are represented in Table 3.

Drug interactions associated with rilpivirine use can be categorized as agents that should never be coadministered with rilpivirine, agents requiring adjustment or monitoring, and agents that require no intervention. An abbreviated list of drug interactions associated with rilpivirine use is found in Table 2. There are a number of agents that should not be coadministered with rilpivirine. The most notable are proton pump inhibitors, due to increases in gastric $\mathrm{pH}$ and reduction in rilpivirine concentrations. ${ }^{16}$ Additionally, anticonvulsants and antimycobacterials with CYP-inductive effects should be avoided with rilpivirine, because concentrations of rilpivirine may be decreased. ${ }^{16}$ Macrolide antibiotics like clarithromycin and erythromycin should be avoided due to CYP inhibition. ${ }^{16,68}$ Where possible, alternative macrolide agents like azithromycin should be utilized. Antacids and $\mathrm{H} 2$ receptor antagonists should be used with caution, and administration should be spaced out from rilpivirine administration. ${ }^{16,68}$ While there have not been studies evaluating this interaction, medications that prolong the QTc interval should be used with caution because there is a risk of QTc prolongation with rilpivirine at higher doses. It is unclear if the use of rilpivirine and a QTc prolonging medication would result in an accentuated risk of QTc prolongation. Electrocardiographic monitoring of high-risk patients using QTc prolonging medications and rilpivirine may be considered.

Of note, a significant interaction occurs when switching from efavirenz to rilpivirine. The long half-life of efavirenz may be concerning when switching to rilpivirine, because efavirenz concentrations persist for weeks after drug discontinuation and efavirenz induces the metabolism of rilpivirine. Pharmacokinetic differences were observed in a study of 20 healthy patients who received rilpivirine for 14 days followed by a washout period and subsequently received efavirenz for 14 days followed by rilpivirine for 28 days. ${ }^{69}$ All rilpivirine pharmacokinetic exposures were significantly lower after efavirenz exposure compared with rilpivirine use prior to efavirenz exposure. ${ }^{69} \mathrm{~A}$ switch study was performed in HIV-infected patients with undetectable viral loads using efavirenz-tenofovir-emtricitabine who sought to switch to rilpivirine-tenofovir-emtricitabine due to intolerance. ${ }^{70}$ The average trough plasma rilpivirine concentration was $52 \mathrm{ng} / \mathrm{mL}$ two weeks after switching therapy. ${ }^{70}$ For weeks $4-12$, the average trough plasma rilpivirine concentration was $66-84 \mathrm{ng} / \mathrm{mL} .^{70}$ In the clinical trials that evaluated the efficacy of rilpivirine, trough concentrations were $50-80 \mathrm{ng} / \mathrm{mL}$. ${ }^{1,3}$ While the trough concentrations in the switch study did not fall below this range, it is unclear if patients may be at risk for subtherapeutic concentrations of rilpivirine in the presence of other medications that affect rilpivirine concentrations, like $\mathrm{H} 2$ receptor antagonists or antacids. Of note, the efavirenz

Table 2 Pharmacokinetic characteristics

\begin{tabular}{|c|c|c|c|}
\hline Pharmacokinetic parameter & Rilpivirine & Tenofovir & Emtricitabine \\
\hline Oral bioavailability & Unknown & $25 \%$ & $93 \%$ \\
\hline Protein binding & $99.7 \%$ & $<0.7 \%$ & $<4 \%$ \\
\hline Elimination pathway & Hepatic & Renal & Renal \\
\hline Elimination half-life & Approximately 48 hours & Approximately I7 hours & Approximately 10 hours \\
\hline Predominant elimination pathway & Hepatic (CYP 3A) & Renal & Renal \\
\hline
\end{tabular}

Abbreviation: CYP, cytochrome P450. 
Table 3 Abbreviated drug interactions associated with rilpivirine

\begin{tabular}{|c|c|}
\hline Concomitant medication & Comments \\
\hline \multicolumn{2}{|c|}{ Medications that should not be coadministered with rilpivirine } \\
\hline $\begin{array}{l}\text { Anticonvulsants: carbamazepine, oxcarbazepine, } \\
\text { phenobarbital, phenytoin }\end{array}$ & $\begin{array}{l}\text { Strong CYP enzymatic induction may result in significant decreases in rilpivirine plasma } \\
\text { concentrations }\end{array}$ \\
\hline Antimycobacterials: rifampin and rifabutin & $\begin{array}{l}\text { Strong CYP enzymatic induction may result in significant decreases in rilpivirine plasma } \\
\text { concentrations. } \\
\text { Rilpivirine AUC, } C_{\min } \text { and } C_{\max } \text { decreased by } 46 \%, 49 \% \text {, and } 35 \% \text {, respectively, when used } \\
\text { concomitantly with rifabutin. } \\
\text { Rilpivirine AUC, } C_{\min } \text { and } C_{\max } \text { decreased by } 80 \%, 89 \% \text {, and } 69 \% \text {, respectively, when used } \\
\text { concomitantly with rifampin }\end{array}$ \\
\hline Proton pump inhibitors & $\begin{array}{l}\text { Changes in gastric } \mathrm{pH} \text { leading to subsequent decreases in rilpivirine exposure. } \\
\text { Rilpivirine } \mathrm{AUC} \text { and } \mathrm{C}_{\min } \text { decrease by } 40 \% \text { and } 33 \% \text {, respectively, when omeprazole } \\
\text { coadministered }\end{array}$ \\
\hline Efavirenz & $\begin{array}{l}\text { AUC of rilpivirine decreased by } 70 \% \text { in presence of efavirenz. } \\
\text { Important pharmacokinetic implications when switching from efavirenz to rilpivirine. May extend } \\
\text { to other NNRTI agents with persistent inductive effects on CYP system }\end{array}$ \\
\hline $\begin{array}{l}\text { Macrolide antibiotics: clarithromycin, } \\
\text { erythromycin }\end{array}$ & $\begin{array}{l}\text { May result in increased exposure to rilpivirine through inhibition of CYP } 3 \mathrm{~A} \text {. Consider use of } \\
\text { azithromycin, where possible }\end{array}$ \\
\hline \multicolumn{2}{|c|}{ Medications requiring adjustment or added monitoring when coadministered with rilpivirine } \\
\hline Ketoconazole & $\begin{array}{l}\text { Inhibition of } C Y P 3 A \text { isoenzymes may result in increased rilpivirine plasma concentrations } \\
\left(C_{\max } \text { and } C_{\min } \text { increased by } 30 \% \text { and } 76 \% \text {, respectively) and decreased concentrations of }\right. \\
\text { ketoconazole }\left(C_{\max } \text { and } C_{\min } \text { decreased by } 15 \% \text { and } 65 \% \text {, respectively). }\right. \\
\text { Monitor for breakthrough fungal infections. Other antifungal agents may be evaluated for usage }\end{array}$ \\
\hline $\mathrm{H} 2$ receptor antagonists & $\begin{array}{l}\text { Use with caution because } \mathrm{H} 2 \text { receptor antagonists increase the gastric } \mathrm{pH} \text {. } \\
\mathrm{H} 2 \text { receptor antagonists and rilpivirine should be spaced out. } \mathrm{H} 2 \text { receptor antagonists should be } \\
\text { administered } 12 \text { hours before rilpivirine or } 4 \text { hours after rilpivirine ingestion }\end{array}$ \\
\hline Antacids & Antacids change gastric $\mathrm{pH}$. Administer antacids 2 hours before or 4 hours after rilpivirine dose. \\
\hline Methadone & $\begin{array}{l}\text { AUC of R- and S-methadone decreased by } 16 \% \text { in the presence of rilpivirine. } C_{\text {min }} \text { of both R- and } \\
\text { S-methadone decreased by about } 21 \% \text {. Monitor for signs of methadone withdrawal. May need to } \\
\text { adjust dose }\end{array}$ \\
\hline Antiarrhythmics & Potential for decreased concentrations of rilpivirine \\
\hline Medications known to prolong QT interval & Potential pharmacodynamic interaction with other QT prolonging medications \\
\hline Tenofovir & $\begin{array}{l}\text { Rilpivirine pharmacokinetics virtually unchanged in presence of tenofovir. Tenofovir } \\
\text { pharmacokinetics slightly elevated }\left(C_{\min } 24 \% \text { higher }\right) \text { in presence of rilpivirine. Consider } \\
\text { monitoring serum creatinine }\end{array}$ \\
\hline \multicolumn{2}{|c|}{ Medications requiring no dosage adjustment or intervention } \\
\hline Didanosine & Coadminister on an empty stomach \\
\hline Darunavir/ritonavir & $\begin{array}{l}\text { No dosage adjustment recommended. However, rilpivirine AUC is } 130 \% \text { higher with darunavir/ } \\
\text { ritonavir coadministration }\end{array}$ \\
\hline Lopinavir/ritonavir & $\begin{array}{l}\text { No dosage adjustment recommended. However, rilpivirine AUC is } 52 \% \text { higher with lopinavir/ } \\
\text { ritonavir coadministration }\end{array}$ \\
\hline Atorvastatin & No dosage adjustment recommended \\
\hline Acetaminophen & No dosage adjustment recommended \\
\hline Ethinyl estradiol & Subtle increases in $A \cup C, C_{\min }$ and $C_{\max }$ of ethinyl estradiol. No dosage adjustment necessary \\
\hline Sildenafil & No dosage adjustment recommended \\
\hline
\end{tabular}

Abbreviations: $A \cup C$, area under the concentration-time curve; $C_{\max }$, peak plasma concentration; $C_{\text {min }}$, trough plasma concentration; $C Y P$, cytochrome P450; NNRTI, non-nucleoside reverse transcriptase inhibitor.

concentrations remained above the $\mathrm{IC}_{50}$ for 4 weeks after the switch to rilpivirine occurred.

\section{Place in therapy}

The vast majority of clinical data with fixed-dose combination rilpivirine-tenofovir-emtricitabine have emerged from studies performed in the treatment-naïve patient population. As a result, this is the patient population that will most commonly be prescribed fixed-dose combination rilpivirinetenofovir-emtricitabine. To date, fixed-dose combination rilpivirine-tenofovir-emtricitabine has not been adequately studied in patients with existing antiretroviral drug resistance and is not officially indicated for use in treatmentexperienced patients. Despite this, rilpivirine may remain a sensitive therapy option in patients who have developed resistance to first-generation NNRTI agents and future study 
may demonstrate a role for rilpivirine in this group. Patients with transmitted NNRTI resistance may be a particular target population for fixed-dose combination rilpivirine-tenofoviremtricitabine.

The role of fixed-dose combination rilpivirine-tenofoviremtricitabine has not been assessed in each antiretroviral treatment guideline to date. The United States Department of Health and Human Services antiretroviral treatment guidelines have recommended that fixed-dose combination rilpivirinetenofovir-emtricitabine be considered as an alternative option for NNRTI-based therapy. Fixed-dose combination efavirenztenofovir-emtricitabine continues to be the preferred NNRTI for most patients, unless specific adverse event risks, such as pregnancy, preclude its use. Because patients who entered clinical study with a baseline viral load $>100,000$ copies $/ \mathrm{mL}$ were more likely to experience virologic failure with rilpivirine-based therapy, caution is recommended when using fixed-dose combination rilpivirine-tenofovir-emtricitabine in patients with high viral loads ( $>100,000$ copies/mL). Equally concerning is the higher rate of drug resistance exhibited after rilpivirine failure and the subsequent rate of phenotypic cross-resistance to etravirine, nevirapine, and efavirenz. Virologic failure and subsequent drug resistance with rilpivirine appears to limit future NNRTI therapy options more uniformly when compared with patients who experience virologic failure and drug resistance to other NNRTI agents.

When compared with the current standard of care, the advantage of rilpivirine in clinical study was its safety and tolerability. As a result, this is likely to be the greatest advantage of rilpivirine over existing therapy options, especially efavirenz. Given that efavirenz-based therapy continues to be the preferred NNRTI therapy option as per treatment guidelines, it is likely that fixed-dose combination rilpivirinetenofovir-emtricitabine will fill an important need as transitional therapy in patients prescribed fixed-dose combination efavirenz-tenofovir-emtricitabine who are unable to tolerate adverse effects attributed to efavirenz. For example, if patients experience persistent central nervous system toxicity with fixed-dose combination efavirenz-tenofovir-emtricitabine, fixed-dose combination rilpivirine-tenofovir-emtricitabine is likely to represent an attractive therapy option, particularly when their HIV viral load is $\leq 100,000$ copies $/ \mathrm{mL}$ at the time of the switch. To date, there has been a small study to evaluate the safety and efficacy of switching patients from fixed-dose combination efavirenz-tenofovir-emtricitabine to fixed-dose combination rilpivirine-tenofovir-emtricitabine. Data from this study have demonstrated reliable efficacy and improved tolerability after the switch, but more data are necessary to ensure virologic efficacy after the switch, particularly when considering the pharmacokinetic drug interaction that occurs between efavirenz and rilpivirine within the first couple weeks after the switch. ${ }^{69}$ Another important advantage of fixed-dose combination rilpivirinetenofovir-emtricitabine over fixed-dose combination efavirenz-tenofovir-emtricitabine is safety during pregnancy. Efavirenz is a pregnancy category $\mathrm{D}$ agent while rilpivirine is a pregnancy category $\mathrm{B}$ agent. ${ }^{35,71,72}$ When considering therapy for female patients of childbearing potential, fixeddose combination rilpivirine-tenofovir-emtricitabine may be a preferred therapy option under certain circumstances.

\section{Summary}

Single-tablet regimens represent an enormous step forward in the objective of providing safe, effective, and conveniently dosed antiretroviral therapy to HIV-infected patients. Fixed-dose combination rilpivirine-tenofoviremtricitabine has been shown to provide a high degree of virologic efficacy and safety in clinical study and will be an important therapy option for some patients. When compared with the current standard of care, concerns over increased risk for virologic failure and drug resistance, particularly among patients with high viral loads, will limit its widespread use in the HIV population. Due to the unique resistance profile of rilpivirine relative to first-generation NNRTI agents, fixed-dose combination rilpivirine-tenofovir-emtricitabine may prove to have an important therapeutic role for patients with existing antiretroviral resistance, but clinical studies are currently lacking in this patient population. For now, the most likely role for fixed-dose combination rilpivirine-tenofoviremtricitabine is to provide an important role as a switch therapy for patients experiencing adverse effects on the current NNRTI standard of care.

\section{Disclosure}

$\mathrm{CDM}$ is on the speakers bureau for Bristol Myers Squibb. The authors otherwise report no conflicts of interest in this work.

\section{References}

1. Cohen CJ, Andrade-Villanueva J, Clotet B, et al. Rilpivirine versus efavirenz with two background nucleoside or nucleotide reverse transcriptase inhibitors in treatment-naive adults infected with HIV-1 (THRIVE): a phase 3, randomised, non-inferiority trial. Lancet 2011;378(9787):229-237.

2. Lennox JL, Dejesus E, Berger DS, et al. Raltegravir versus efavirenz regimens in treatment-naive HIV-1-infected patients: 96-week efficacy, durability, subgroup, safety, and metabolic analyses. J Acquir Immune Defic Syndr. 2010;55(1):39-48. 
3. Molina JM, Cahn P, Grinsztejn B, et al. Rilpivirine versus efavirenz with tenofovir and emtricitabine in treatment-naive adults infected with HIV-1 (ECHO): a phase 3 randomised double-blind active-controlled trial. Lancet. 2011;378(9787):238-246.

4. Maggiolo F, Airoldi M, Kleinloog HD, et al. Effect of adherence to HAART on virologic outcome and on the selection of resistanceconferring mutations in NNRTI- or PI-treated patients. HIV Clin Trials. 2007;8(5):282-292.

5. Paterson DL, Swindells S, Mohr J, et al. Adherence to protease inhibitor therapy and outcomes in patients with HIV infection. Ann Intern Med. 2000;133(1):21-30.

6. Bucher HC, Wolbers M, Porter K. 2010 guidelines for antiretroviral treatment of HIV from the International AIDS Society-USA Panel. JAMA. 2010;304(17):1897; author reply 1897-1898.

7. Mordant C, Schmitt B, Pasquier E, et al. Synthesis of novel diarylpyrimidine analogues of TMC278 and their antiviral activity against HIV-1 wild-type and mutant strains. Eur J Med Chem. 2007;42(5):567-579.

8. [No authors listed]. A once-daily combination tablet (Atripla) for HIV. Med Lett Drugs Ther. 2006;48(1244):78-79.

9. [No authors listed]. European Commission approves Atripla. AIDS Patient Care STDS. 2008;22(1):87-88.

10. Arribas JR, Pozniak AL, Gallant JE, et al. Tenofovir disoproxil fumarate, emtricitabine, and efavirenz compared with zidovudine/lamivudine and efavirenz in treatment-naive patients: 144-week analysis. J Acquir Immune Defic Syndr. 2008;47(1):74-78.

11. Pozniak AL, Gallant JE, DeJesus E, et al. Tenofovir disoproxil fumarate, emtricitabine, and efavirenz versus fixed-dose zidovudine/lamivudine and efavirenz in antiretroviral-naive patients: virologic, immunologic, and morphologic changes - a 96-week analysis. J Acquir Immune Defic Syndr. 2006;43(5):535-540.

12. [No authors listed]. Atripla approval in Europe. AIDS Patient Care STDS. 2006;20(12):887.

13. Bera E, McCausland K, Nonkwelo R, Mgudlwa B, Chacko S, Majeke B. Birth defects following exposure to efavirenz-based antiretroviral therapy during pregnancy: a study at a regional South African hospital. AIDS. 2010;24(2):283-289.

14. Schouten JT, Krambrink A, Ribaudo HJ, et al. Substitution of nevirapine because of efavirenz toxicity in AIDS clinical trials group A5095. Clin Infect Dis. 2010;50(5):787-791.

15. [No authors listed]. FDA notifications. Complera approved. AIDS Alert. 2011;26(11):130-131.

16. Miller V, de Bethune MP, Kober A, et al. Patterns of resistance and cross-resistance to human immunodeficiency virus type 1 reverse transcriptase inhibitors in patients treated with the nonnucleoside reverse transcriptase inhibitor loviride. Antimicrob Agents Chemother. 1998;42(12):3123-3129.

17. Complera ${ }^{\circledR}$ (package insert). Foster City, CA: Gilead Sciences; 2011.

18. Janssen PA, Lewi PJ, Arnold E, et al. In search of a novel antiHIV drug: multidisciplinary coordination in the discovery of 4-[[4-[[4-[(1E)-2-cyanoethenyl]-2,6-dimethylphenyl]amino]-2pyrimidinyl]amino]benzonitrile (R278474, rilpivirine). J Med Chem. 2005;48(6):1901-1909.

19. Lansdon EB, Brendza KM, Hung M, et al. Crystal structures of HIV-1 reverse transcriptase with etravirine (TMC125) and rilpivirine (TMC278): implications for drug design. J Med Chem. 2010;53(10): 4295-4299.

20. Das K, Clark AD Jr, Lewi PJ, et al. Roles of conformational and positional adaptability in structure-based design of TMC125-R165335 (etravirine) and related non-nucleoside reverse transcriptase inhibitors that are highly potent and effective against wild-type and drug-resistant HIV-1 variants. J Med Chem. 2004;47(10):2550-2560.

21. Antinori A, Zaccarelli M, Cingolani A, et al. Cross-resistance among nonnucleoside reverse transcriptase inhibitors limits recycling efavirenz after nevirapine failure. AIDS Res Hum Retroviruses. 2002;18(12):835-838.
22. Deeks SG, Barditch-Crovo P, Lietman PS, et al. Safety, pharmacokinetics, and antiretroviral activity of intravenous 9-[2-(R)-(Phosphonomethoxy) propyl]adenine, a novel anti-human immunodeficiency virus (HIV) therapy, in HIV-infected adults. Antimicrob Agents Chemother. 1998;42(9):2380-2384.

23. Suo Z, Johnson KA. Selective inhibition of HIV-1 reverse transcriptase by an antiviral inhibitor, (R)-9-(2-Phosphonylmethoxypropyl)adenine. J Biol Chem. 1998;273(42):27250-27258.

24. Ying C, De Clercq E, Neyts J. Lamivudine, adefovir and tenofovir exhibit long-lasting anti-hepatitis B virus activity in cell culture. JViral Hepat. 2000;7(1):79-83.

25. Brenner B, Turner D, Oliveira M, et al. A V106M mutation in HIV-1 clade $\mathrm{C}$ viruses exposed to efavirenz confers cross-resistance to nonnucleoside reverse transcriptase inhibitors. AIDS. 2003;17(1):F1-F5.

26. Richman DD. Antiretroviral activity of emtricitabine, a potent nucleoside reverse transcriptase inhibitor. Antivir Ther. 2001;6(2):83-88.

27. Hazen R, Lanier ER. Relative anti-HIV-1 efficacy of lamivudine and emtricitabine in vitro is dependent on cell type. J Acquir Immune Defic Syndr. 2003;32(3):255-258.

28. Korba BE, Schinazi RF, Cote P, Tennant BC, Gerin JL. Effect of oral administration of emtricitabine on woodchuck hepatitis virus replication in chronically infected woodchucks. Antimicrob Agents Chemother. 2000;44(6):1757-1760

29. Goebel F, Yakovlev A, Pozniak AL, et al. Short-term antiviral activity of TMC278 - a novel NNRTI - in treatment-naive HIV-1-infected subjects. AIDS. 2006;20(13):1721-1726.

30. Molina JM, Peytavin G, Perusat S, et al. Pharmacokinetics of emtricitabine, didanosine and efavirenz administered once-daily for the treatment of HIV-infected adults (pharmacokinetic substudy of the ANRS 091 trial). HIV Med. 2004;5(2):99-104.

31. Wang LH, Begley J, St Claire RL III, Harris J, Wakeford C, Rousseau FS. Pharmacokinetic and pharmacodynamic characteristics of emtricitabine support its once daily dosing for the treatment of HIV infection. AIDS Res Hum Retroviruses. 2004;20(11):1173-1182.

32. Van Heeswijk RH, Hoetelmans R, Kestens D, et al. The pharmacokinetic (PK) interaction between famotidine and $\mathrm{TMC} 278$, a next generation non-nucleoside reverse transcriptase inhibitor (NNRTI), in HIV-negative volunteers. Abstract TUPDB01 presented at the 4th IAS Conference on HIV Pathogenesis, Treatment and Prevention, July 22-25, 2007, Sydney, Australia.

33. Hawkins T, Veikley W, St Claire RL III, Guyer B, Clark N, Kearney BP. Intracellular pharmacokinetics of tenofovir diphosphate,carbovir triphosphate, and lamivudine triphosphate in patients receiving triple-nucleoside regimens. J Acquir Immune Defic Syndr. 2005;39(4):406-411.

34. Van Heeswijk RH, Hoetelmans R, Aharchi F, et al. The pharmacokinetic (PK) interaction between atorvastatin (AVS) and TMC278, a nextgeneration non-nucleoside reverse, transcriptase inhibitor (NNRTI). Presented at the 11th European AIDS Conference, October 24-27, 2007, Madrid, Spain.

35. Edurant ${ }^{\circledR}$ (Package insert). Titusville, NJ: Janssen; 2011

36. Van Heeswijk RH, Hoetelmans R, Kestens D, et al. The pharmacokinetic interaction between ketoconazole and TMC278, an investigational non-nucleoside reverse transcriptase inhibitor, in healthy HIV-negative subjects. Abstract TUPE0087 presented at the 16th International AIDS Conference, August 13-18, 2006, Toronto, Canada.

37. Kearney BP, Yale K, Shah J, Zhong L, Flaherty JF. Pharmacokinetics and dosing recommendations of tenofovir disoproxil fumarate in hepatic or renal impairment. Clin Pharmacokinet. 2006;45(11):1115-1124.

38. Airoldi M, Zaccarelli M, Bisi L, et al. One-pill once-a-day HAART: a simplification strategy that improves adherence and quality of life of HIV-infected subjects. Patient Prefer Adherence. 2010;4:115-125.

39. Dejesus E, Young B, Morales-Ramirez JO, et al. Simplification of antiretroviral therapy to a single-tablet regimen consisting of efavirenz, emtricitabine, and tenofovir disoproxil fumarate versus unmodified antiretroviral therapy in virologically suppressed HIV-1-infected patients. J Acquir Immune Defic Syndr. 2009;51(2):163-174. 
40. Fisher M, Moyle GJ, Shahmanesh M, et al. A randomized comparative trial of continued zidovudine/lamivudine or replacement with tenofovir disoproxil fumarate/emtricitabine in efavirenz-treated HIV-1-infected individuals. J Acquir Immune Defic Syndr. 2009;51(5):562-568.

41. Crauwels H, van Heeswijk RPG, Stevens T, et al. Relative bioavailability of a concept pediatric formulation of TMC278, an investigational NNRTI. Abstract THPE0158 presented at the XVIIIth International AIDS Conference, July 18-23, 2010, Vienna, Austria.

42. Fatkenheuer G, Duvivier C, Rieger A, et al. Lipid profiles for etravirine versus efavirenz in treatment-naive patients in the randomized, double-blind SENSE trial. J Antimicrob Chemother. 2012;67(3):685-690.

43. Rhoads MP, Lanigan J, Smith CJ, Lyall EG. Effect of specific ART drugs on lipid changes and the need for lipid management in children with HIV. J Acquir Immune Defic Syndr. 2011;57(5):404-412.

44. Wilkin A, Pozniak AL, Morales-Ramirez J, et al. Long-term efficacy, safety, and tolerability of rilpivirine (RPV, TMC278) in HIV Type 1-infected antiretroviral-naive patients: week 192 results from a Phase IIb randomized trial. AIDS Res Hum Retroviruses. October 17, 2011. [Epub ahead of print.]

45. Viread ${ }^{\mathrm{TM}}$ (package insert). Foster City, CA: Gilead Sciences; 2012.

46. Blaas S, Schneidewind A, Gluck T, Salzberger B. Acute renal failure in HIV patients with liver cirrhosis receiving tenofovir: a report of two cases. AIDS. 2006;20(13):1786-1787.

47. Hynes P, Urbina A, McMeeking A, Barisoni L, Rabenou R. Acute renal failure after initiation of tenofovir disoproxil fumarate. Ren Fail. 2007;29(8):1063-1066.

48. Zimmermann AE, Pizzoferrato T, Bedford J, Morris A, Hoffman R, Braden G. Tenofovir-associated acute and chronic kidney disease: a case of multiple drug interactions. Clin Infect Dis. 2006;42(2):283-290.

49. Verhelst D, Monge M, Meynard JL, et al. Fanconi syndrome and renal failure induced by tenofovir: a first case report. Am J Kidney Dis. 2002;40(6):1331-1333.

50. Cooper RD, Wiebe N, Smith N, Keiser P, Naicker S, Tonelli M. Systematic review and meta-analysis: renal safety of tenofovir disoproxil fumarate in HIV-infected patients. Clin Infect Dis. 2010;51(5): 496-505.

51. Irizarry-Alvarado JM, Dwyer JP, Brumble LM, Alvarez S, Mendez JC. Proximal tubular dysfunction associated with tenofovir and didanosine causing Fanconi syndrome and diabetes insipidus: a report of 3 cases. AIDS Read. 2009;19(3):114-121.

52. Karras A, Lafaurie M, Furco A, et al. Tenofovir-related nephrotoxicity in human immunodeficiency virus-infected patients: three cases of renal failure, Fanconi syndrome, and nephrogenic diabetes insipidus. Clin Infect Dis. 2003;36(8):1070-1073.

53. Coca S, Perazella MA. Rapid communication: acute renal failure associated with tenofovir: evidence of drug-induced nephrotoxicity. Am J Med Sci. 2002;324(6):342-344.

54. Gallant JE, Parish MA, Keruly JC, Moore RD. Changes in renal function associated with tenofovir disoproxil fumarate treatment, compared with nucleoside reverse-transcriptase inhibitor treatment. Clin Infect Dis. 2005;40(8):1194-1198.

55. Gupta SK. Tenofovir-associated Fanconi syndrome: review of the FDA adverse event reporting system. AIDS Patient Care STDS. 2008;22(2):99-103.

56. Peyriere H, Reynes J, Rouanet I, et al. Renal tubular dysfunction associated with tenofovir therapy: report of 7 cases. J Acquir Immune Defic Syndr. 2004;35(3):269-273.

HIV/AIDS - Research and Palliative Care

\section{Publish your work in this journal}

HIV/AIDS - Research and Palliative Care is an international, peerreviewed open-access journal focusing on advances in research in HIV, its clinical progression and management options including antiviral treatment, palliative care and public healthcare policies to control viral spread. The journal welcomes original research, basic science,
57. McComsey GA, Kitch D, Daar ES, et al. Bone mineral density and fractures in antiretroviral-naive persons randomized to receive abacavirlamivudine or tenofovir disoproxil fumarate-emtricitabine along with efavirenz or atazanavir-ritonavir: Aids Clinical Trials Group A5224s, a substudy of ACTG A5202. J Infect Dis. 2011;203(12):1791-1801.

58. Cahn P. Emtricitabine: a new nucleoside analogue for once-daily antiretroviral therapy. Expert Opin Investig Drugs. 2004;13(1):55-68.

59. Gish RG, Leung NW, Wright TL, et al. Dose range study of pharmacokinetics, safety, and preliminary antiviral activity of emtricitabine in adults with hepatitis B virus infection. Antimicrob Agents Chemother. 2002;46(6):1734-1740.

60. Emtriva $^{\mathrm{TM}}$ (package insert). Foster City, CA: Gilead Sciences; 2007.

61. Nelson M, Schiavone M. Emtricitabine (FTC) for the treatment of HIV infection. Int J Clin Pract. 2004;58(5):504-510.

62. Kuritzkes DR. Drug resistance in HIV-1. Curr Opin Virol. 2011;1(6): 582-589.

63. Das K, Bauman JD, Clark AD Jr, et al. High-resolution structures of HIV-1 reverse transcriptase/TMC278 complexes: strategic flexibility explains potency against resistance mutations. Proc Natl Acad Sci USA. 2008;105(5):1466-1471.

64. Haubrich RH, Riddler SA, DiRienzo AG, et al. Metabolic outcomes in a randomized trial of nucleoside, nonnucleoside and protease inhibitor-sparing regimens for initial HIV treatment. AIDS. 2009;23(9):1109-1118.

65. Wittkop L, Gunthard HF, de Wolf F, et al. Effect of transmitted drug resistance on virological and immunological response to initial combination antiretroviral therapy for HIV (EuroCoord-CHAIN joint project): a European multicohort study. Lancet Infect Dis. 2011;11(5):363-371.

66. Rimsky L, Vingerhoets J, Van Eygen V, et al. Genotypic and phenotypic characterization of HIV-1 isolates obtained from patients on rilpivirine therapy experiencing virologic failure in the phase $3 \mathrm{ECHO}$ and THRIVE studies: 48-week analysis. JAcquir Immune Defic Syndr. 2012;59(1):39-46.

67. Miller CD, Crain J, Tran B, Patel N. Rilpivirine: a new addition to the anti-HIV-1 armamentarium. Drugs Today (Barc). 2011;47(1):5-15.

68. Hughes CA, Robinson L, Tseng A, MacArthur RD. New antiretroviral drugs: a review of the efficacy, safety, pharmacokinetics, and resistance profile of tipranavir, darunavir, etravirine, rilpivirine, maraviroc, and raltegravir. Expert Opin Pharmacother. 2009;10(15):2445-2466.

69. Crauwels H, Vingerhoets J, Ryan R, Witek J, Anderson D. Pharmacokinetic parameters of once-daily TMC278 following administration of EFV in healthy volunteers. Abstract 630 presented at the 18th Conference on Retroviruses and Opportunistic Infections. February 27-March 2, 2011, Boston, MA.

70. Mills A, Cohen C, Dejesus E, et al. Switching from efavirenz/emtricitabine/tenofovir disoproxil fumarate (EFV/FTC/TDF) single tablet regimen (STR) to emtricitabine/rilpivirine/tenofovir disoproxil fumarate (FTC/RPV/TDF) STR in virologically suppressed, HIV-1 infected subjects. Abstract $\mathrm{H} 2-794 \mathrm{c}$ presented at the 51st Interscience Conference on Antimicrobial Agents and Chemotherapy (ICAAC). September 17-20, 2011, Chicago, IL.

71. Panel on Antiretroviral Guidelines for Adults and Adolescents. Guidelines for the use of antiretroviral agents in HIV-1-infected adults and adolescents. Department of Health and Human Services. November 17, 2011. Available from: http://www.aidsinfo.nih.gov/ ContentFiles/AdultandAdolescentGL.pdf. Accessed March 8, 2012.

72. Atripla ${ }^{\mathrm{TM}}$ (package insert). New York, NY: Bristol-Myers Squibb and Gilead Sciences; 2011.

\section{Dovepress}

clinical \& epidemiological studies, reviews \& evaluations, expert opinion \& commentary, case reports \& extended reports. The manuscript management system is completely online and includes a very quick and fair peer-review system. Visit http://www.dovepress.com/ testimonials.php to read real quotes from published authors. 